\title{
Effect of substrate defects on commensurate-incommensurate transitions of adsorbed layers
}

\author{
J. Villain \\ Département de Recherche Fondamentale, Laboratoire de Diffraction Neutronique, \\ Centre d'Etudes Nucléaires de Grenoble, 85X, 38041 Grenoble Cedex, France
}

(Reçu le 25 février 1980, révisé le 9 avril, accepté le 11 avril 1980)

\begin{abstract}
Résumé. - On propose une théorie à température nulle de la transition Commensurable-Incommensurable d'une couche adsorbée sur un substrat de symétrie hexagonale contenant des défauts. La symétrie hexagonale est conservée au voisinage d'une transition continue, en désaccord avec le résultat de Bak et al. pour un substrat idéal mais en accord avec l'expérience pour le krypton adsorbé sur graphite. La loi de puissance observée expérimentalement pourrait résulter d'un métissage entre un comportement à la Frank-Van der Merwe et une loi linéaire inobservable à cause d'effets de taille finie.
\end{abstract}

\begin{abstract}
The effect of certain substrate defects on the Commensurate-Incommensurate transition of adsorbed layers is studied theoretically at zero temperature in the case of hexagonal substrate. Hexagonal symmetry is preserved near a continuous transition, in contrast with the ideal substrate case studied by Bak et al., but in agreement with experiments on krypton films on graphite. The apparent power law observed experimentally is interpreted as resulting from a crossover from a Frank-Van der Merwe behaviour to a linear law which is never reached because of finite size effects.
\end{abstract}

1. Introduction. - Bak, Mukamel, Villain and Wentowska [1] (BMVW) proposed a theory for the Commensurate-Incommensurate (C-I) transition. The theory could be applied, for instance, to a monolayer physisorbed on an ideal substrate of square or hexagonal symmetry at zero temperature. The theory predicted either a first order transition (possibly without change of symmetry) or a continuous transition with lower symmetry in the incommensurate phase. However, experiments on krypton on graphite [2-5] are consistent with a continuous transition without loss of symmetry. In the present work the BMVW theory is extended to the case of an infinite substrate containing randomly distributed impurities. Effect of temperature has been considered in another paper [6].

2. The BMVW model. - In the BMVW model [1], the incommensurate phase was regarded as a network of walls separating domains. The system is essentially commensurate inside each domain. For instance in the case of krypton monolayers on graphite there are three types of domains A, B, C separated by walls which have three allowed directions $[7,8]$ at $120^{\circ}$ from each other. For definiteness attention will be focussed on this case in this communication. In the case of hexagonal symmetry, walls form a regular honeycomb network (Fig. 1a) at zero temperature. The energy can be written as a sum of three terms : i) A pressure dependent contribution proportional to the total wall length $L$. ii) A term proportional to the number $2 v$ of wall intersections. iii) An interaction between walls which is an exponential function of the distance $l \sqrt{3}$ between walls as explained by various authors [9-13]

$$
W=\mu L+2 \Lambda v+C^{\prime} L \exp -\kappa l \sqrt{3} .
$$

The C-I transition is triggered by the variation of $\mu$ with pressure. Coefficients $C$ and $\Lambda$ can be assumed to be pressure independent.

The results crucially depend on the sign of $\Lambda$ [1]. For $\Lambda<0$ the C-I transition is first order. For $\Lambda>0$ the C-I transition is continuous but the distorted configuration of figure $1 b$ has a lower energy than 


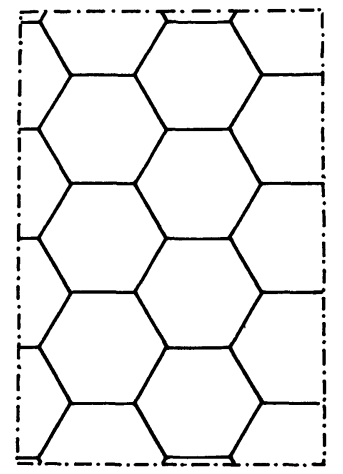

a

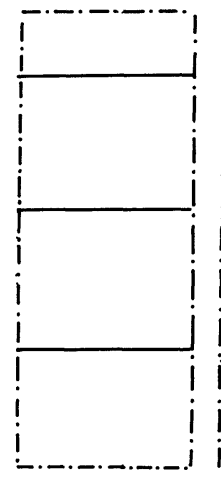

b

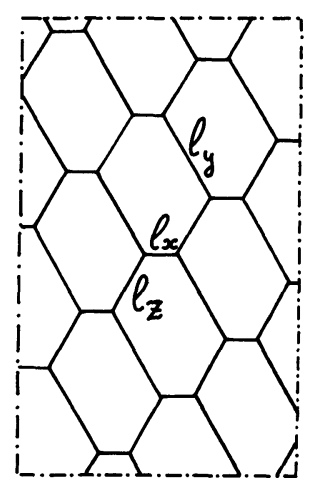

C
Fig. 1. - Possible wall configurations at $T=0$ on an ideal substrate. a) Regular honeycomb network. $b$ ) Striped structure predicted by BMVW for $\Lambda>0$. c) Semi-regular honeycomb network.

the hexagonally symmetric configuration of figure $1 a$, at least near the transition. Its energy is :

$$
W=\mu L+C L \exp -\kappa d
$$

where $d$ is the distance between walls. If $\mathcal{N}$ is the total area of the sample, $d=\mathcal{N} / L$. The coefficient $C$ is generally different from $C^{\prime}$ [1]. Energy (2) is lower than (1) because the positive $\Lambda$ term is absent and the exponentiel repulsion is small for large distances, i.e. near the transition.

More generally it is of interest to consider the semiregular honeycomb network displayed by figure $1 c$, though in no case it was found to minimize the energy.

3. Effect of impurities. - The honeycomb lattice has the remarkable property that it can be distorted without change of $L$ and $v$ by increasing or decreasing all sides of any hexagon $\mathrm{i}$ by the same amount $\delta l_{\mathrm{i}}$ as shown by figure $2 a$. Each variable $\delta l_{\mathrm{i}}$ may be considered as a soft degree of freedom, which only acts on the last term of (1). This term is exponentially small for large distances between walls. The number of soft degrees of freedom is equal to the number $v$ of hexagons, or more precisely [6] to $(v+1)$ in the case of periodic boundary conditions. When all hexagons are modified the walls form an irregular honeycomb network (Fig. $2 b$ ).

We will now argue that certain substrate defects have precisely the effect of distorting the honeycomb network. These defects may be impurities, or small terraces, or even perhaps steps. Grain boundaries and dislocations are excluded because they spoil the general description in terms of walls and domains.

Defects create a random potential which locally favours A or B or C domains. Therefore an appropriate distortion of domains (Fig. 2a) produces an energy gain. The energy gain per domain is expected to be proportional to the square root of the domain area $\mathcal{N} / v$, as argued by Imry and Ma [14] and by Lee
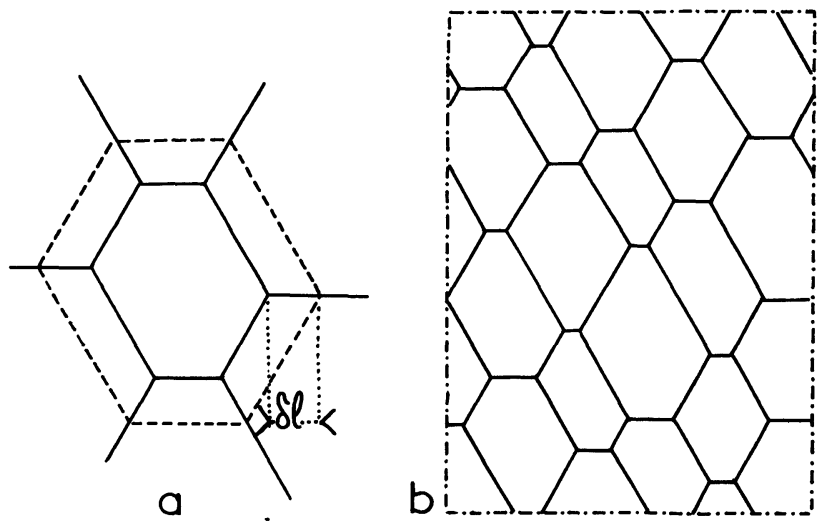

Fig. 2. - a) A transformation of the wall network. It modifies neither $L$ nor $v . b$ ) Irregular honeycomb network obtained from figure $1 c$ when applying this transformation to all hexagons.

and Rice [15] (see also references therein). This result comes essentially from the central limit theorem since the area change per domain is also expected to be proportional to $\mathcal{N} / v$. The average energy gain resulting from domain distortion is for $v$ domains :

$$
W_{\text {def }}=-w_{\mathrm{d}} v \sqrt{\mathcal{N} / v}=-w_{\mathrm{d}} \mathcal{N} \sqrt{v / \mathcal{N}}
$$

where the coefficient $w_{\mathrm{d}}$ depends on the nature and concentration of defects. It has to be positive since $W_{\text {def }}$ is an energy gain. Formula (3) implies serious approximations which will be discussed in the next section. We find it convenient to investigate its consequences first.

The total ground state energy is the sum of $W_{\text {def }}$ and the terms considered in section 2. The wall configuration may be assumed to result from the distortion of a semi-regular hexagonal network characterized by hexagon sides $l_{x}, l_{y}, l_{z}$ (Figs. $1 c$ and $2 b$ ). The corresponding ground state energy can be written as :

$$
\begin{aligned}
W=\mu L-w_{\mathrm{d}} \mathcal{N} & \sqrt{v / \mathcal{N}}+2 \Lambda v+ \\
& +L\left[C_{x} \mathrm{e}^{-\kappa l_{x}}+C_{y} \mathrm{e}^{-\kappa l_{y}}+C_{z} \mathrm{e}^{-\kappa l_{z}}\right]
\end{aligned}
$$

where $C_{x}, C_{y}, C_{z}$ are functions of $l_{x} / l_{z}$ and $l_{y} / l_{z} . L$ and $v$ are easily seen to be given by :

$$
\begin{aligned}
& L=v\left(l_{x}+l_{y}+l_{z}\right) \\
& v=\frac{1}{2} \mathcal{N}\left(l_{x} l_{y}+l_{y} l_{z}+l_{z} l_{x}\right)^{-1} .
\end{aligned}
$$

For a given value of $L, W_{\text {def }}$ is easily seen to be minimum for $l_{x}=l_{y}=l_{z}=l$. Thus, the second term of (4) favours hexagonal symmetry. On the other hand the 3rd term favours symmetry lowering [1] if 
$\Lambda$ is positive. However this term is proportional to $\mathcal{N} / l^{2}$ while $W_{\text {def }}$ is proportional to $\mathcal{N} / l$. $W_{\text {def }}$ is therefore larger for large $l$. Therefore, hexagonal symmetry is preserved near a continuous C-I transition, in contrast with the BMVW result. Detailed calculations confirm this argument.

4. Discussion. - 1) Formula (3) implies a number of approximations which will be discussed now. Firstly, the defects produce a shift of the chemical potential $\mu$ which is not relevant for our purpose and has therefore not been included in the formulae. This shift has been observed in certain experiments [16].

2) A more serious point is that substrate defects can deviate walls from their easy directions [15]. Such wall distortions produce an energy gain even in the case of parallel walls (Fig. $1 b$ ) in contradiction with formula (3). However, this energy gain is expected to vanish when the wall stiffness goes to infinity. Therefore, expression (3) should be correct if walls are sufficiently stiff.

3) The coefficient $w_{\mathrm{d}}$ has been treated as a constant in section 3. However, it may depend somewhat on the ratios $l_{x} / l_{z}$ and $l_{y} / l_{z}$. Therefore these ratios may eventually be different from 1 in the ground state although this very asymmetric configuration (Fig. 1c) does not seem plausible. Only the BMVW configuration of figure $1 b$ is strictly excluded near a continuous C-I transition if $w_{\mathrm{d}}$ is positive.

4) Expression (3) is the leading term of the defect contribution. Unfortunately it is proportional to $L$ like the first term of the energy (4). The behaviour of $l$ near the transition depends on higher order terms [1] as in the Landau theory of order-disorder transitions. If we are lucky, the leading higher order term may be the third term of expression (4), but this term may well be dominated by some contribution arising from defects. For instance, in the case of ideally rigid walls, the transformation of figure $2 a$ may only change the area by a quantized amount proportional to $l$. The associated energy quantum should be proportional to $\sqrt{l}$ according to the central limit theorem. This introduces a correction proportional to $\mathrm{Nl}^{-3 / 2}$ into expression (4). This correction dominates the last two terms.

5) Defects can destroy the topology of the honeycomb network, since they can produce dislocations of the Kosterlitz-Thouless type. The importance of these dislocations has been pointed out by Venables et al. [17]. Nevertheless we neglect them. This certainly is a serious approximation, which may be acceptable if dislocations have a very high energy.

5. Theory and experiments. - A precise comparison between theory and experiment seems extremely difficult. In addition to the fundamental difficulties listed in the above discussion, the coefficient $w_{\mathrm{d}}$ of expression (3) can hardly be evaluated since it depends on the nature of defects. The case of krypton monolayers on graphite is of special interest since it has 'been studied very carefully [2-5] and the experimental' data are in disagreement with the BMVW theory. The quantity $1 / l$ defined in section 3 may be identified with the average misfit which has been measured by Leed [3] and X-ray diffraction [5] as a function of the natural misfit which is a linear function of our parameter $-\mu$. We suggest the following, tentative interpretation of the experimental data. There are three regimes.

i) For large values of - $\mu$ the Frank-Van der Merwe theory [18] (FVdM) theory is applicable [3]. This implies that the third term of expression (4) is negligible.

ii) For low values of $-\mu$ the curve $1 / l=f(\mu)$ exhibits a tail which may be attributed to the finite size of the samples.

iii) We suggest the existence of an intermediate region where the third term of expression (4) may not be neglected. Even if $\Lambda$ is positive, hexagonal symmetry is preserved if $w_{\mathrm{d}}$ is large enough. For positive $\Lambda$ the curve $1 / l=f(\mu)$ is less steep than in the FVdM theory. For instance, if the last term of expression (4) is neglected, expressions (4), (5) and (6) yields a linear variation :

$$
1 / l=\left(2 w_{\mathrm{d}}-3 \mu\right) / 4 \Lambda .
$$

The experimental curve [5] is less steep than the FVdM curve, but steeper than expression (7). This indicates that the last term of (4) may not be neglected. Stephens et al. [5] and Fain et al. [19] have fitted their experimental data by a power law with an exponent $1 / 3$. We suggest this fit to be the accidental result of a crossover from the FVdM behaviour to the linear law (7), which is never reached because of finite size effects. Another power law is also possible since $W_{\text {def }}$ may contain a correction to expression (3), as noticed in section 4 . Let this correction be proportional to $\mathcal{N} / l^{1+\eta}$, with a positive coefficient. A negative coefficient would produce a first order transition [1]. $\eta$ should lie between 0 and 1 in order to dominate the third term of expression (4). Formula (7) is replaced by :

$$
1 / l \sim\left(\mu_{\mathrm{c}}-\mu\right)^{1 / \eta} .
$$

The exponent is larger than 1 in contradiction with experiment. Again we attribute this discrepancy to the last term of (4), which cannot be neglected.

The situation described above is not the only possible one. A first order transition is expected for negative $\Lambda$. For positive $\Lambda$ the symmetry lowering predicted by BMVW may occur for appropriate values of $w_{\mathrm{d}}, \Lambda$ and $C$. We have shown that it does not occur for very large $l$, but it is still allowed for 
intermediate values of $l$. Indeed, striped superstructures have been observed for $\mathrm{CO}$ on Co and Pt [21] and for Cs on W and Mo [22].

6. Conclusion. - Substrate defects have been shown to have a drastic influence on C-I transitions. They destroy the symmetry lowering predicted by BMVW for a continuous transition. We have not been able to provide a complete theory, but we have proposed a tentative interpretation of experimental data. Other plausible interpretation rely on long range interactions [6] or restricted area effects [20].
Unfortunately no numerical evaluation of the various effects has been made, so that it is difficult to judge these attempts.

Again we stress that the present work is very preliminary. In particular, thermal effects have been considered in reference [6] for an ideal substrate, and it would be of interest to extend this work when defects are present.

Acknowledgments. - I am grateful to Sam Fain for many informations and explanations on the experimental situation.

\section{References}

[1] Bak, P., Mukamel, D., Villain, J., Wentowska, K., Phys. Rev. B 19 (1979) 1610.

[2] Tномy, A., Duval, X., J. Chim. Phys. 66 (1969) 1966 and 67 (1970) 286, 1101

[3] Chinn, M. D., Fain, S. C., Phys. Rev. Lett. 39 (1977) 146 and J. Physique Colloq. 38 (1977) C4-99.

[4] Larher, Y., J. Chem. Phys. 68 (1978) 2257.

[5] Stephens, P. W., Heiney, P., Birgeneau, R. J., Horn, P. M., Phys. Rev. Lett. 43 (1979) 47.

[6] Villain, J., to be published in Surf. Sci. (1980).

[7] Venables, J. A., Schabes-Retchkiman, P. S., J. Physique Colloq. 38 (1977) C4-105.

[8] Villain, J., in Ordering in strongly fluctuating systems Ed. T. Riste (Plenum Press, New York) 1980, p. 214.

[9] Mc Millan, W. L., Phys. Rev. B 14 (1976) 1496.

[10] BaK, P., EMERY, V., Phys. Rev. Lett. 36 (1976) 978.

[11] Luban, M., Mukamel, D., Shtrikman, S., Phys. Rev. A 10 (1976) 360.
[12] Bruce, A. D., Cowley, R. A., J. Phys. C 11 (1978) 3577, 3591, 3609.

[13] Villain, J., Phys. Rev. Lett. 41 (1978) 36.

[14] Imry, Y., Ma, S. K., Phys. Rev. Lett. 35 (1975) 1399.

[15] LeE, P. A., Rice, T. M., Phys. Rev. B 19 (1979) 3970.

[16] Marti, C., Thorel, P., Croset, B., Le Vide Suppl. 185 (1977) 27.

[17] Venables, J. A., Schabes-Retchkiman, P. S., J. Phys. C 11 (1978) L913.

[18] Frank, F. C., Van der Merwe, J. H., Proc. R. Soc. London 198 (1949) 205

[19] Fain, S. C., ChinN, M. D., Diehl, R. D., to be published in Phys. Rev. B (Comments and addenda) (1980).

[20] PokrovskiI, V. L., TALAPov, A. L.; to appear in Zh. E.T.F. (1980).

[21] Conrad, H., ErTl, G., KüPPERS, J., Surf. Sci. 76 (1978) 323.

[22] Bol'shov, L. A., Napartovich, A. L., Naumovetz, A. G., Fedorus, A. G., Uspekhi Fiz. Nauk 122 (1977) 125. 